\title{
Properties of Different Coronal Streamers
}

\section{S. Parenti}

Centre for Astrophysics, University of Central Lancashire, Preston PR1 2HE, UK Run in Address to Make Minimum Number of Lines

\section{B. J. I. Bromage}

Centre for Astrophysics, University of Central Lancashire, Preston PR1 2HE, $U K$

\section{G. Poletto}

Osservatorio Astrofisico di Arcetri, I-50125 Firenze, Italy

G. Noci

Universita' di Firenze, I-50125 Firenze, Italy

J. C. Raymond

Harvard-Smithsonian Center for Astrophysics, Cambridge, MA 02138, $U S A$

G. E. Bromage

Centre for Astrophysics, University of Central Lancashire, Preston PR1 2HE, UK

\begin{abstract}
In this paper a summary of results from coordinated observations of streamers acquired by the SOHO Coronal Diagnostic Spectrometer (CDS) and UltraViolet Coronograph Spectrometer (UVCS) experiments is presented (Parenti et al. 2000). Data from different altitudes within the solar corona were taken, with the purpose of determining their physical parameters - densities, electron temperatures and element abundances - and their changes over the altitude range between 1.02 and 1.6 $R_{\odot}$. Further UVCS streamer data, taken about two months later are used to compare with the behaviour seen in two different streamers. The streamers resulted to have a different behaviour in temperature and density. The low corona, covered by CDS data, shows little evidence of abundance variation with respect to photospheric values, while, at UVCS altitudes, a depletion of all element abundances is clearly evident.
\end{abstract}




\section{Introduction}

Coronal streamers are prominent structures of the solar corona. Limited to the equatorial regions during the minimum of solar activity, spanning towards the polar region during maximum, they are one of the evident manifestations of solar activity, very well visible on white light and EUV images. Their main morphological and physical properties have been studied for many years, but with the SOHO mission new unknown characteristics have been revealed. From a "Whole Sun Month" campaign, density and temperature profile inside streamers, up to several solar radii, were derived (Gibson at el. 1999). Raymond et al. (1997) obtained absolute element abundances inside streamers, showing a remarkable depletion of all the elements, compared to the photospheric values.

\section{Results and discussion}

An equatorial and a mid-latitude streamer were observed between 1.02 and 1.6 $R_{\odot}$ using CDS and UVCS instruments on board SOHO, on the 8 March 1998. A further mid-latitude streamer was observed in May 1998 with the UVCS only. Profiles of temperature and density v. heliospheric distance showed a good agreement between values derived from the two instruments (Figure 1 and 2). The equatorial streamer appears to be a "quiet streamer". It is less dense than the March mid-latitude streamer and the temperature tends to isothermal value even at low distance. The mid-latitude streamers appear to be "active". The March streamer is not isothermal in the low corona but it is at greater distance. The May streamer is still multithermal at $1.6 \mathrm{R}_{\odot}$. The densities derived from the two instruments show a good agreement, and the decrease in densities with distance can be well reproduced by assuming hydrostatic equilibrium.

Using different methods the $\mathrm{O}$ VI and Fe and other element abundances were obtained from the UVCS data. (Table 1).

Most of the abundances from UVCS data show a depletion with respect to the photosphere, confirming previous observations (Raymond et al. 1997). Oxygen is more depleted in the equatorial streamer than in the mid-latitude data. The average $\mathrm{Fe} / \mathrm{O}$ ratio of 0.13 derived here is consistent with the 0.11 derived for the in-ecliptic solar wind (Aellig at al. 1999).

Abundances relative to $\mathrm{Fe}$, were instead derived from CDS data. The relative abundances from CDS data show only a slight deviation from photospheric values. Comparing UVCS and CDS results for the few elements in common, there is a clear depletion in the abundances with distance. More detailes of these results may be found in Parenti et al. paper.

\section{References}

Aellig, M.R. et al. 1999, JGR, 104, 24, 769

Gibson, S.E. et al. 1999, JGR, 104, 9691

Parenti, S. et al A\&A in press

Raymond, J.C. et al. 1997, Solar Phys, 175 


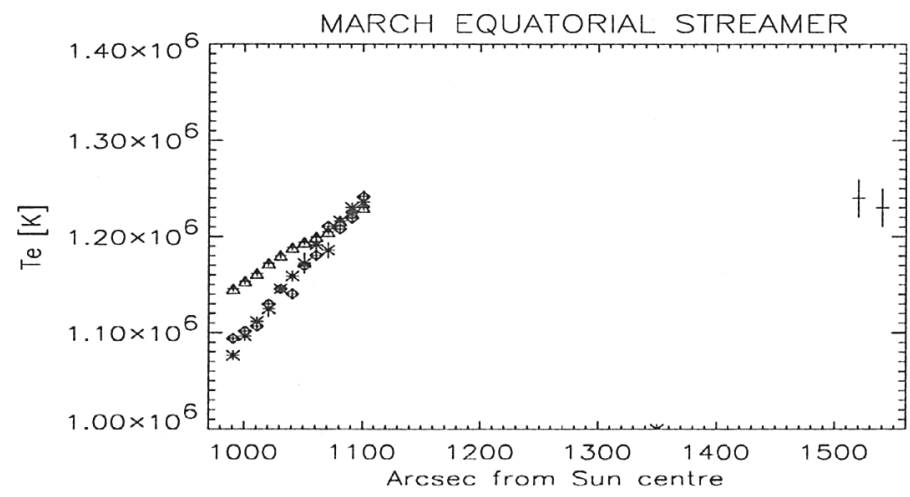

Figure 1. Temperature $v$. distance from the Sun centre for the March equatorial streamers. The triangles are the temperatures from CDS Fe XIII 348.183/ Fe X 345.723; asterisks are the temperature from CDS Fe $\mathrm{XI} 352.662$ / Fe X 345.723; diamonds are the temperatures from CDS Fe XII 364.467/ Fe XI 352.662; crosses are the temperatures from UVCS Fe XIII 510/ Fe X 1028.

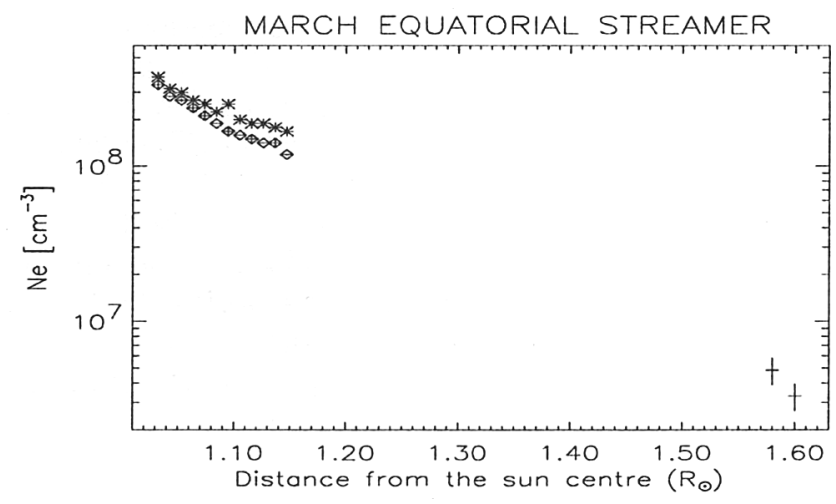

Figure 2. Density profile $v$. distance from the Sun centre for the March equatorial streamer (top). The diamonds represent the density from CDS Si IX 349.9/341.9, the asterisks the density from CDS Si X $356 / 347$ and the triangles the density from UVCS.

Table 1. Absolute abundances from UVCS

\begin{tabular}{|c|c|c|c|c|c|c|c|c|}
\hline Region & $\mathrm{O}$ & $\mathrm{Mg}$ & $\mathrm{Al}$ & $\mathrm{Si}$ & $S$ & $\mathrm{Ar}$ & $\mathrm{Ca}$ & $\mathrm{Fe}$ \\
\hline & & 7.58 & 6.47 & & 7.21 & 6.65 & 6.36 & 7.51 \\
\hline & & & & & 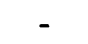 & & & 7.51 \\
\hline & 8.00 & 7.10 & 6.00 & 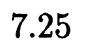 & - & 0 & - & 7.51 \\
\hline & 8.30 & - & - & 7.2 & - & 0 & - & 7.34 \\
\hline & 8.20 & 7.00 & 5.90 & 7.2 & - & & - & 7.34 \\
\hline & 8.40 & 7.20 & 6.25 & 7.30 & 7.21 & 6.58 & 6.36 & 7.20 \\
\hline
\end{tabular}

